Dhaka Univ. J. Sci. 61(1): 125-129, 2013 (January)

\title{
Effect of Nd Substitution on the Microstructural and Dielectric Properties of Polycrystalline $\mathrm{Ca}\left(\mathrm{Ti}_{0.5} \mathrm{Fe}_{0.5}\right) \mathrm{O}_{3}$
}

\author{
M. R. Shah ${ }^{1 *}$, M. R. Amin ${ }^{2}$ and A. K. M. Akther Hossain ${ }^{3}$ \\ ${ }^{1}$ Department of Physics, Primeasia University, Banani, Dhaka-1213, Bangladesh \\ ${ }^{2}$ Department of Theoretical Physics, University of Dhaka, Dhaka-1000, Bangladesh \\ ${ }^{3}$ Department of Physics, Bangladesh University of Engineering and Technology, Dhaka-1000, Bangladesh
}

Received on 05.06.2012. Accepted for Publication on 04. 11. 2012

\begin{abstract}
Neodymium substituted calcium iron titanate having the general formula $\mathrm{Ca}_{1-\mathrm{x}} \mathrm{Nd}_{\mathrm{x}}\left(\mathrm{Ti}_{0.5} \mathrm{Fe}_{0.5}\right) \mathrm{O}_{3}$ were prepared by the standard solid state reaction technique at relatively higher temperature $(1473 \mathrm{~K})$. X-ray diffraction (XRD) and optical microscopy are used to carry out the structural analysis and surface morphology, respectively. The XRD analysis confirms that all compositions are single phase orthorhombic in structure. The lattice parameters and the average grain size are found to decrease but the density to increase with the increase in $\mathrm{Nd}$ content. The dielectric constant $\left(\varepsilon^{\prime}\right)$, dielectric loss $(\tan \delta)$ and ac conductivity $\left(\sigma_{\mathrm{ac}}\right)$ are studied at room temperature as a function of frequency and compositions. The room temperature $\varepsilon$ is found to decrease with the increase in frequency and $\mathrm{Nd}$ content. On the other hand, the tan $\delta$ and $\sigma_{\mathrm{ac}}$ are observed to increase with the increase in frequency and decrease with the increase in Nd content.
\end{abstract}

Keywords: Dielectric properties, Microstructure, Solid state reaction, X-ray diffraction.

\section{Introduction}

The complex perovskite with general formula $\left(\mathrm{ABO}_{3}\right)$ exhibiting high dielectric constant are widely used for detectors, multi-layer ceramic capacitors, sensors, actuators, power transmission devices, memory devices, high energy storage devices, pyroelectric detectors and other electronic devices ${ }^{1-2}$. Generally, perovskite materials containing $\mathrm{Pb}$ compound such as PMN, PST, PLZT exhibit outstanding physical properties, such as giant dielectric constants, exceptionally large electrostrictive coefficients and electrooptic constants which are considered potential properties for use in modern electronic devices ${ }^{3-4}$. However, these $\mathrm{Pb}$ based perovskite compounds are environment-unfriendly because $\mathrm{PbO}$ is toxic. Researchers have now been researching for $\mathrm{Pb}$ free perovskite materials whose physical properties will be comparable with those of $\mathrm{Pb}$ containing compounds $^{5-6}$. Among these $\mathrm{Pb}$ free materials, $\mathrm{CaTiO}_{3}$ (CTO) based solid solutions are considered as environmentfriendly with similar performance as $\mathrm{Pb}$ based perovskite. These materials are widely used due to their interesting mechanical, chemical and ferroelectric properties. However these properties of CTO may be improved by the substitution of different types of dopants so that it can be employed in wide range of devices, such as: multilayer capacitors, thermistors, sensors, wireless communications. The substitution can be done either at the $\mathrm{Ca}$ and/or Ti site of $\mathrm{CTO}^{7-8}$. It has been reported that oxide materials that exhibit simultaneous ferromagnetic and ferroelectric characteristics are known as multiferroics have attracted considerable attention of researcher ${ }^{9-10}$. However, it is a very rare case in nature that both magnetic and electric polarization coexist in one substance. Therefore, multiferroics become the subject of intensive investigations due to their potential applications in the emerging field of spintronics, data-storage media, multiple-state memories etc. As an $\mathrm{ABO}_{3}$-type ferroelectric oxide, CTO has become one of the most promising systems for multiferroics research. Its structural characteristics determine that $3 \mathrm{~d}$ transition metal Ti can be easily substituted to produce ferromagnetism. Therefore, $\mathrm{Fe}$ is substituted for Ti because Fe has a strong resemblance to the $\mathrm{Ti}$ ion in size and valence and in the present study, $50 \%$ doping of $\mathrm{Fe}$ is done to study the multiferroics properties of CTO. Doped with rare earth oxide is also an effective method to vary the ferroelectric and magnetic properties of materials. Several kinds of rare earth oxide such as: $\mathrm{La}_{2} \mathrm{O}_{3}, \mathrm{Y}_{2} \mathrm{O}_{3}, \mathrm{Sm}_{2} \mathrm{O}_{3}$ have been used to improve further dielectric properties of CTO ceramics, ${ }^{8,11-12}$. However, the research on $\mathrm{Nd}_{2} \mathrm{O}_{3}$ doped CTO ceramics have not been reported in the literature. The radius of $\mathrm{Nd}^{3+}$ is very close to the radius of the A-sites $\left(\mathrm{Ca}^{2+}\right)$, it is therefore possible for $\mathrm{Nd}^{3+}$ to enter into the A-sites of CTO perovskite and affect the properties of CTO ceramics. In view of this, $\mathrm{Nd}_{2} \mathrm{O}_{3}$ is selected as the dopant of $\mathrm{Ca}_{1-\mathrm{x}} \mathrm{Nd}_{\mathrm{x}}\left(\mathrm{Ti}_{0.5} \mathrm{Fe}_{0.5}\right) \mathrm{O}_{3}$ (CNTFO) (where $\mathrm{x}=0.0,0.1,0.3$ and 0.5 ) and the effects of $\mathrm{Nd}_{2} \mathrm{O}_{3}$ substitution on the phase composition, microstructure and dielectric properties of CNTFO were investigated.

\section{Experimental Details}

\section{Synthesis of CNTFO ceramic obtained by solid state reaction}

Polycrystalline CNTFO compositions were prepared by the standard solid state reaction technique. In this technique, the powder of $\mathrm{CaCO}_{3}(99.9 \%), \mathrm{Nd}_{2} \mathrm{O}_{3}(99.9 \%), \mathrm{TiO}_{2}(99.9 \%)$

\footnotetext{
* Corresponding Author: E-mail: hirubd@yahoo.com
} 
and $\mathrm{Fe}_{2} \mathrm{O}_{3}(99.9 \%)$ were used as raw materials. These powders were stoichiometrically mixed thoroughly by ball milling in distilled water media for $10 \mathrm{~h}$ to obtain a homogeneous powder. Afterward, the slurry was dried and the mixed powders were calcined at $1223 \mathrm{~K}$ for $12 \mathrm{~h}$ in air. The calcined powders were re-milled for $10 \mathrm{~h}$ and then dried. Finally, the dried powders were grounded and then disk-shaped pellets of diameter of $10 \mathrm{~mm}$ and thickness 1-2 $\mathrm{mm}$ were prepared from the fine powders under uniaxial pressure. The pellets were sintered at $1473 \mathrm{~K}$ for $5 \mathrm{~h}$. During sintering, the heating and cooling rates were maintained at $0.16 \mathrm{~K} / \mathrm{s}$ and $0.08 \mathrm{~K} / \mathrm{s}$, respectively.

\section{Characterizations}

The phase-purity and crystal structure of the compositions were investigated by the $\mathrm{XRD}$ with $\mathrm{CuK}_{\alpha}$ radiation at room temperature. The measured bulk density ' $\rho_{\mathrm{B}}$ ' of the compositions was determined using the expression: $\rho_{B}=(W$ $\times \rho) /\left(\mathrm{W}-\mathrm{W}^{\prime}\right)$, where $\mathrm{W}$ and $\mathrm{W}^{\prime}$ are the weights of the compositions in air and water, respectively, and $\rho$ is the density of water at room temperature. The theoretical density ' $\rho_{\text {th }}$ ' was calculated using the expression: $\rho_{\text {th }}=$ $\left\{10^{3} \times 4 \mathrm{M}_{\mathrm{A}} /\left(\mathrm{N}_{\mathrm{A}} \mathrm{abc}\right)\right\} \mathrm{kg} / \mathrm{m}^{3}$, where $N_{A}$ is the Avogadro's number, $M_{A}$ is the molecular weight, $a, b$ and $c$ are the lattice parameters for the orthorhombic unit cell. The porosity ' $\mathrm{P}$ ' was calculated from the relation: $\mathrm{P}(\%)=$ $\left\{100\left(\rho_{\mathrm{th}}-\rho_{\mathrm{B}}\right) / \rho_{\mathrm{th}}\right\}$.

The surface morphology of sintered polished pellet was studied by a high resolution optical microscope (Olympus DP-70) and the average grain size was determined by the linear intercept technique ${ }^{13}$. In order to measure the dielectric properties, gold electrodes were deposited on both sides of the pellets and then a gold wire was attached on each electrode with silver paste. The frequency dependence of the capacitance and the dielectric loss were measured by LCR meter at room temperature within the frequency range $10 \mathrm{~Hz}$ to $32 \mathrm{MHz}$. The dielectric constant was calculated from the equation: $\varepsilon^{\prime}=(\mathrm{Cd}) /\left(\varepsilon_{0} \mathrm{~A}\right)$, where $C$ is the capacitance (Farad), $\varepsilon_{0}$ is the permittivity in free space, $A$ is the area $\left(\mathrm{m}^{2}\right)$ of the electrode and $d$ is the thickness $(\mathrm{m})$ of the pellet. The ac conductivity was calculated using the dielectric data (obtained at room temperature) from the relation: $\sigma_{\mathrm{ac}}=\varepsilon^{\prime} \varepsilon_{0} \omega \tan \delta$, where $\omega$ is the angular frequency and $\tan \delta$ is the dielectric loss.

\section{Results and Discussion}

\section{Crystal structure, lattice parameters and density}

The XRD pattern for various CNTFO is shown in Fig.1. The XRD pattern indicates that all compositions are single phase perovskite and no second phases are detected. It implies that $\mathrm{Nd}^{3+}$ cations have entered into crystalline lattice structure to form a homogeneous solid solution ${ }^{14}$. The XRD patterns are in good agreement with orthorhombic structure ${ }^{15}$. It is also evident that the diffraction peaks of the compositions are shifted to the higher angle with the increase in $\mathrm{Nd}$ content, this is due to smaller ionic radius of $\mathrm{Nd}^{3+}(1.27 \AA)$ than that of $\mathrm{Ca}^{2+}(1.34 \AA)$ which causes the decrease in interplaner spacing between the lattice ${ }^{14}$.

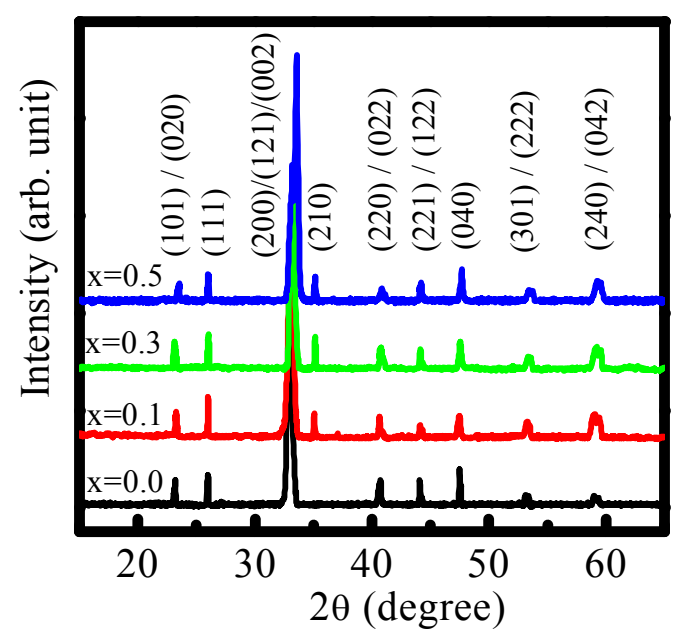

Fig. 1. X-ray diffraction pattern for various CNTFO sintered at $1473 \mathrm{~K}$.

The lattice parameters $a, b$ and $c$ for each compositions of CNTFO plotted as a function of $\mathrm{Nd}$ content are shown in Fig.2. It is observed from Fig.2 that the lattice parameters decrease with the increase in $\mathrm{Nd}$ content. This is due to $\mathrm{Nd}^{3+}$ $(1.27 \AA)$ has a smaller ionic radius than $\mathrm{Ca}^{2+}(1.34 \AA)$ and the substitution of $\mathrm{A}$ site with an ion having smaller ionic radii than the host ion will lead to the shrinkage of the lattice parameters $^{16}$.

The effect of $\mathrm{Nd}$ on the density and porosity of CNTFO is shown in Fig.3. The $\rho_{B}$ increases with the increase in $\mathrm{Nd}$ content. On the other hand, $\mathrm{P}$ of the compositions has opposite behavior. The increase of $\rho_{B}$ with the increase in $\mathrm{Nd}$ content may be attributed to the difference in atomic weight and density of initial and substituted cations, [the atomic weight and density of $\mathrm{Nd}\left(144.24 \mathrm{amu}, 7.00 \times 10^{3}\right.$ $\left.\left.\mathrm{kg} / \mathrm{m}^{3}\right)>\mathrm{Ca}\left(40.00 \mathrm{amu}, 1.55 \times 10^{3} \mathrm{~kg} / \mathrm{m}^{3}\right)\right]^{17}$. The $\rho_{\text {th }}$ increases with the increase in $\mathrm{Nd}$ content because the molecular weight of each composition increases significantly with the substitution of $\mathrm{Nd}$ for $\mathrm{Ca}$. It is also noticed from Fig. 3 that the $\rho_{\text {th }}$ is larger in magnitude compared to the corresponding $\rho_{\mathrm{B}}$. This is due to the existence of pores which are formed and developed during the preparation of compositions or the sintering process ${ }^{17}$. 


\section{Microstructure}

The optical micrographs of various CNTFO sintered at 1473 $\mathrm{K}$ are shown in Fig. 4. The substitution of $\mathrm{Nd}$ has significant effect on the grain size of the compositions and the sintered CNTFO compositions become dense with the increase in $\mathrm{Nd}$ content. It is seen from the Fig. 4 that the average grain size for all the compositions gradually decreases from $4.41 \mu \mathrm{m}$ to $0.56 \mu \mathrm{m}$ with the increase in $\mathrm{Nd}$ content. This result suggests that the incorporation of $\mathrm{Nd}_{2} \mathrm{O}_{3}$ can limit grain growth in the CNTFO. This behavior is believed to be caused by the grain-boundary pinning force of $\mathrm{Nd}_{2} \mathrm{O}_{3}$, which depressed the migration of CNTFO grain boundaries during the sintering process ${ }^{18}$. The decrease of grain size can also be interpreted as the suppression of oxygen vacancies due to charge compensation mechanism, which results in slower oxygen ions motion and, consequently, lower grain growth rate ${ }^{19}$.

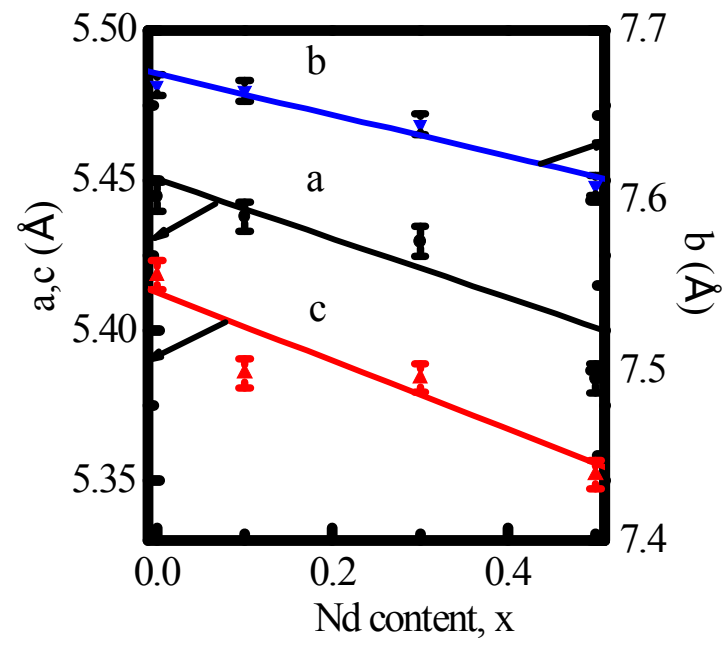

Fig. 2. Variation of lattice parameters with $\mathrm{Nd}$ content of various CNTFO sintered at $1473 \mathrm{~K}$.

\section{Frequency dependence of dielectric properties}

The frequency dependence of dielectric properties measured at room temperature for all compositions is shown in the Fig. 5. It is clear from the Fig. 5(a) that $\varepsilon^{\prime}$ has larger value in the lower frequency region and remains almost constant up to a certain frequency and thereafter decreases rapidly with the increase in frequency.

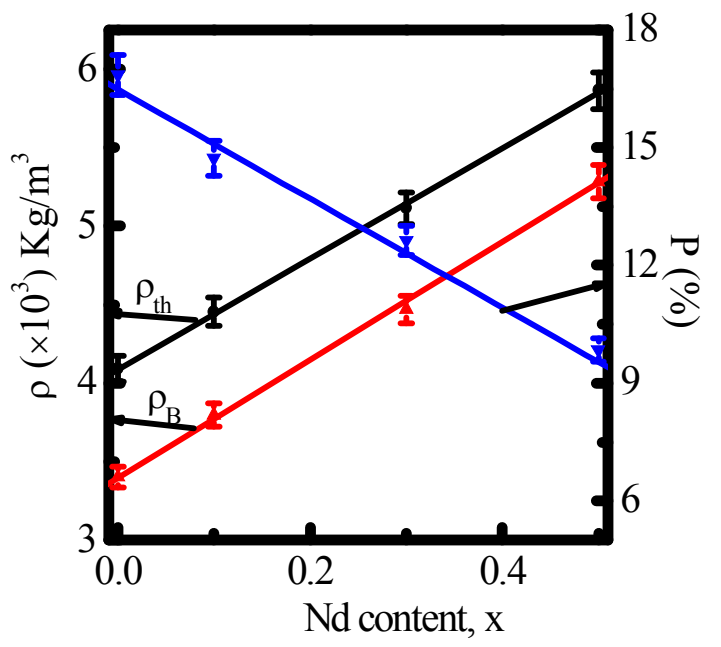

Fig. 3. Variation of density and porosity with $\mathrm{Nd}$ content of various CNTFO sintered at $1473 \mathrm{~K}$.

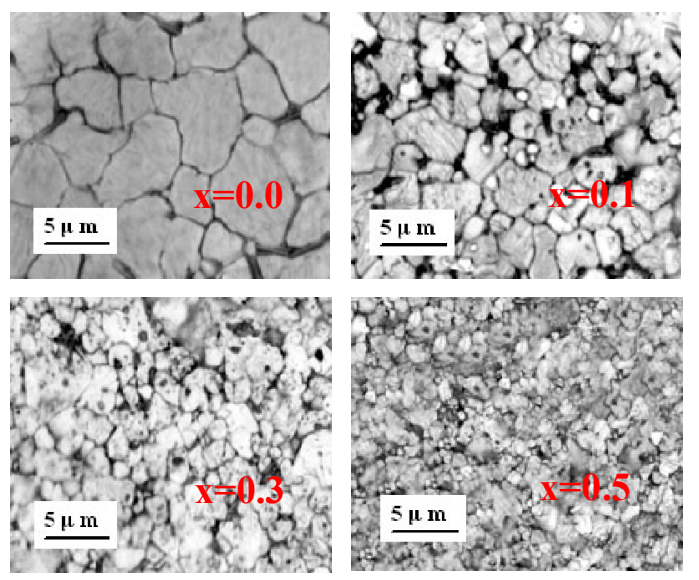

Fig. 4. The optical micrographs of various CNTFO sintered at $1473 \mathrm{~K}$.

The large values of $\varepsilon^{\prime}$ at low frequencies in case of ionic crystals are due to voids, dislocations and other defects. However, in the case of ceramic materials (ferrite and ferroelectric) the large values of $\varepsilon^{\prime}$ have been attributed to the effect of heterogeneity of the compositions like pores and layered structures ${ }^{20}$. The large values of $\varepsilon^{\prime}$ at lower frequencies may also be attributed to the dipoles resulting from changes in valence states of cations and space charge polarization. 


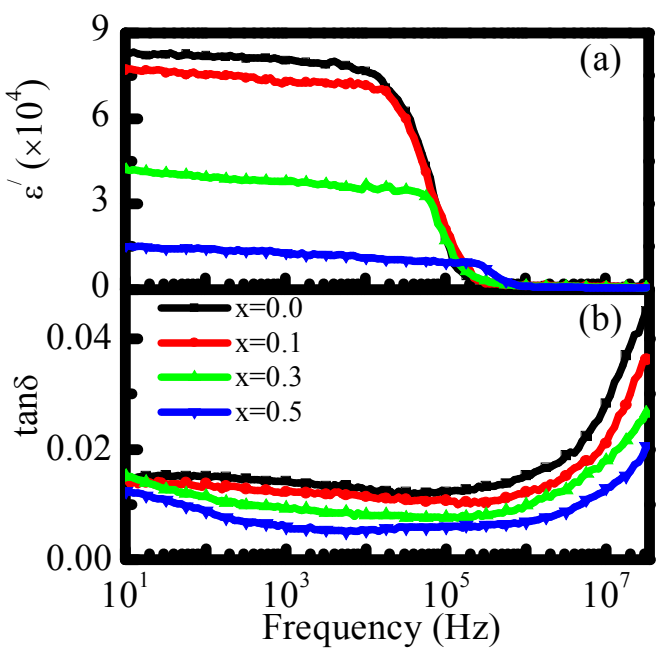

Fig. 5. Frequency dependence of (a) dielectric constant $\left(\varepsilon^{\prime}\right)$ and (b) dielectric loss $(\tan \delta \square)$ of various CNTFO sintered at $1473 \mathrm{~K}$

At higher frequencies, the values of $\varepsilon^{\prime}$ remain independent of frequency, since the inability of electric dipoles to reorient itself to the applied electric field. These frequency independent values of $\varepsilon$ are known as static values of the dielectric constant and these static values in the present compositions are observed beyond $13 \mathrm{kHz}$ to $2.5 \mathrm{MHz}$ (depending upon the composition).

The dielectric loss arises when the polarization lags behind the applied electric field and is caused by the impurities and imperfections in the crystal lattice ${ }^{21}$. Fig. 5(b) shows the variation of $\tan \delta$ with frequency for various compositions. The $\tan \delta$ is minimum at lower frequency region and it increases with the increase in frequency. Such behavior may be explained on the ground that at lower frequency, the dipoles which are formed due to impurities or inhomogeneous structure are able to follow the frequency of the applied electric field; so small energy is required by the dipole to orient itself for polarization resulting in low loss of energy. But with the increase in frequency, the dipoles lag behind the frequency of the applied electric field and therefore, more energy is required for dipole orientation. So, the loss is high at higher frequency.

\section{Composition dependence of dielectric properties}

The composition dependence of $\varepsilon^{\prime}$ and $\tan \delta$ is shown in Fig. 6. It is noticed from Fig. 6 (a) that the values of $\varepsilon$ decrease with the increase in Nd content. This can be explained on the basis of Maxwell-Wagner theory. According to this theory the dielectric constant is directly proportional to the grain size of the samples ${ }^{22}$. In the present study, it is found that the grain size of the compositions decrease with the increase in $\mathrm{Nd}$ content (Fig. 4). The decrease in grain size leads to the decrease in polarizability of atoms in the structure, which results in a decrease in $\varepsilon^{\prime}$.

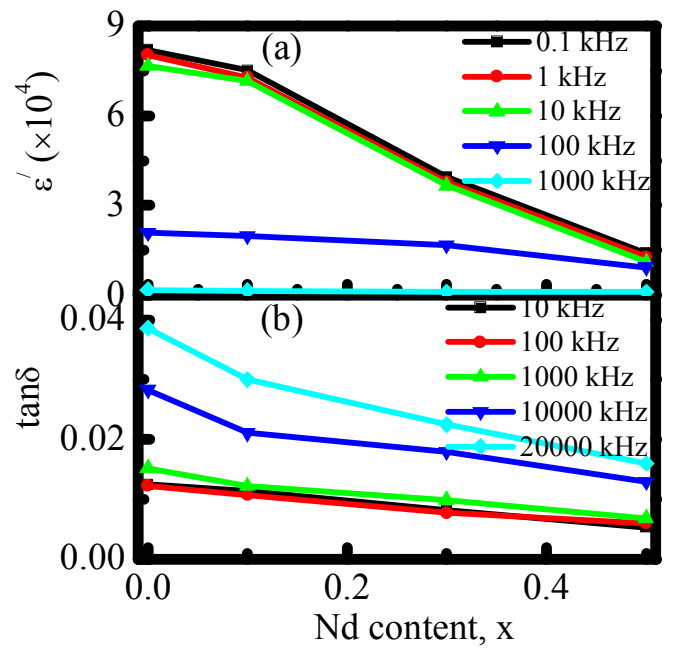

Fig. 6. Composition dependence of (a) dielectric constant $(\varepsilon)$ and (b) dielectric loss ( $\tan \delta$ ) of various CNTFO sintered at $1473 \mathrm{~K}$.

The $\tan \delta$ is found to decrease with the increase in $\mathrm{Nd}$ content as shown in Fig. 6 (b). This may be primarily attributed to the decrease in dielectric constant with the increase in $\mathrm{Nd}$ content as well as increase in frequency. In general high permittivity materials acquire higher losses. Careful observation of the Fig. 5 reveals that the loss is minimum for $\mathrm{x}=0.5$ and maximum for $\mathrm{x}=0.0$ composition. It is obvious as $\varepsilon^{\prime}$ is the lowest for $\mathrm{x}=0.5$ and the highest for $\mathrm{x}=0.0$ composition.

\section{Frequency and Composition dependence of ac conduc- tivity}

Fig. 7 shows the variation of $\sigma_{\mathrm{ac}}$ with $\mathrm{Nd}$ content at different frequencies. This figure indicates that $\sigma_{a c}$ increases with the increase in frequency of the applied electric field, as $\sigma_{\mathrm{ac}}$ is directly proportional to the frequency ${ }^{23}$. The increase of $\sigma_{a c}$ with frequency may also be explained by the hopping of charge carriers between $\mathrm{Ti}^{4+} \leftrightarrow \mathrm{Ti}^{3+}$ or $\mathrm{Fe}^{3+} \leftrightarrow \mathrm{Fe}^{2+}$ ions as with the increase in frequency of the applied electric field enhances the hoping of charge carriers resulting in an increase in the conduction process ${ }^{24}$. It is also noticed from Fig. 7 that the $\sigma_{\mathrm{ac}}$ decrease with the increase in Nd contents. This can be explained on the basis of average grain size as the grain boundary areas are highly resistive in oxide ceramics. The decreased grain size increases the grain boundary areas or resistance, as in the case of smaller grain, 
the number of grain boundary per unit thickness is higher than that of large grain size ceramics. Hence the resistivity of the compositions increases, which in turn decreases the conductivity of the compositions with $\mathrm{Nd}$ content.

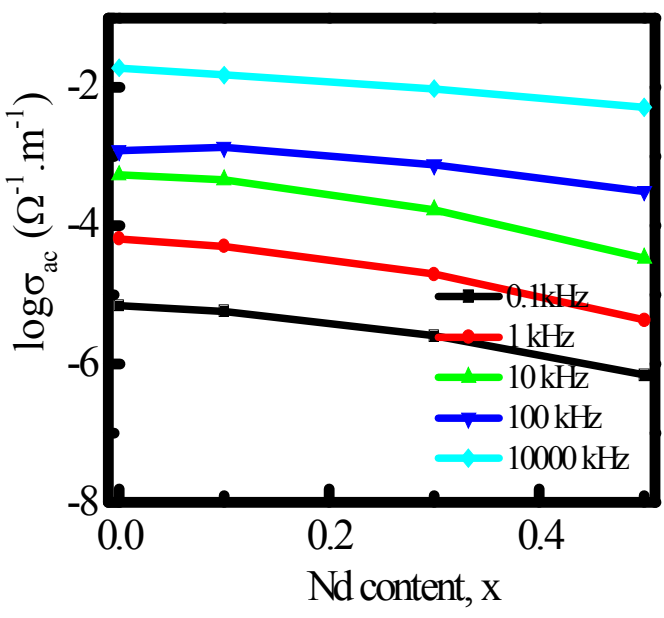

Fig. 7. Compositions dependence of ac conductivity $\left(\sigma_{\mathrm{ac}}\right)$ of various CNTFO sintered at $1473 \mathrm{~K}$.

\section{Conclusions}

The polycrystalline CNTFO are successfully synthesized by the standard solid state reaction technique. The XRD pattern confirms that all the CNTFO compositions are crystallized in a single phase orthorhombic structure. The lattice parameters and average grain sizes are found to decrease, but the density increases with the increase in $\mathrm{Nd}$ content. The $\varepsilon$ is high at lower frequency and remains fairly constant up to a certain frequency and thereafter, it decreases with the increase in frequency. In higher frequency, $\varepsilon^{\prime}$ is independent of frequency due to the inability of electric dipoles to reorient itself to the frequency of the applied electric field. The $\tan \delta \square$ is minimum at lower frequency but maximum at higher frequency. The $\tan \delta \square$ decreases with the increase in $\mathrm{Nd}$ content since the dielectric constant decreases with the increase in $\mathrm{Nd}$ content. The $\sigma_{\mathrm{ac}}$ is found to increase with the increase in frequency.

\section{Acknowledgement}

The authors gratefully acknowledge CASR of Bangladesh University of Engineering and Technology (BUET) for financial support in this research.
1. Jones, R. E., P. D. Mainar, J. O. Olowolafe, J. O. Campbell and C. J. Mogab, 1992. Appl. Phys. Lett. 60, 1022.

2. Cohen, R. E., 2000. J. Phys. Chem. Sol. 61, 139.

3. Mesquita, A., A. Michalowicz, and V. R. Mastelaro, 2012. J. Appl. Phys. 111, 104110.

4. Moore, E. R., P. Ferrari, D. E. D. Droguett, D. Lederman and J. T. Evans, 2012. J. Appl. Phys. 111, 014108.

5. Wang, Y., L. Y. Xiang, W. Dong, and Y. Q. Rui, 2006. Chin. Phys. Lett. 23, 2232.

6. Du, H., W. Zhou, F. Luo, and D. Zhu, 2007. Appl. Phys. Lett. 91, 202907.

7. Chung, C. Y., Y. H. Chang, Y. S. Chang and G. J. Chen, 2004. J. Alloys Compd. 385, 298.

8. Dwivedi, R. K., D. Kumar, O. Parkash, 2001. J. Mater Sci. 36, 3641 .

9. Lin F., D. Jiang, X. Ma, W. Shi, 2008. J. Magn. Magn. Mater. 320, 691.

10. Lin F., D. Jiang, X. Ma, W. Shi, 2008. Physica B 403, 2525.

11. Chourasia, R. and O. P. Shrivastava, 2011. Bull. Mater. Sci., $\mathbf{3 4 , 8 9 .}$

12. Yoon, K. H., W. S. Kim and E. S. Kim, 2003. Mater. Sci. Eng. B 99, 112.

13. Mendelson, M. I., 1969. J. Am. Ceram. Soc. 52, 443.

14. Peng, F., Z. Xu, R. Chu, W. Li, G. Zang and J. Hao, 2010. J. Mater. Chem. Phys. 124, 1065.

15. Cavalcante, L. S., V. S. Marques, J. C. Sczancoski, M. T. Escote, M. R. Joya, J. A. Varela, M. R. M. C. Santos, P. S. Pizani and E. Longo, 2008. J. Chem. Eng. 143, 299.

16. Majumder, S. B., B. Roy, and R. S. Katiyar, 2001. J. Appl. Phys. 90, 2975.

17. Abdeen, A. M., O. M. Hemeda, E. E. Assem, and M. E. Sehly, 2002. J. Magn. Magn. Mater. 238, 75.

18. Jiansirisomboon, S., A. Watcharapasorn, 2008. Curr. Appl. Phys. 8, 48.

19. Kumar, M. and K. L. Yadav, 2007. Appl. Phys. Lett. 91, 112911.

20. Patankar, K. K., S. A. Patil, K. V. Sivakumar, R. P. Mahajan, Y. D. Kolekar and M. B. Kothale, 2000. Mater. Chem. Phys. 65, 97.

21. Singh, N., A. Agarwal and S.Sanghi, 2011. Curr. Appl. Phys. 11, 783.

22. Shah, D. D., P. K. Mehta, M. S. Desai, C. J. Panchal, 2011. J. Alloys Compd. 509, 1800.

23. Mangalaraja, R. V., S. Ananthakumar, P. Manohar, F. D. Gnanam, 2003. Mater. Lett. 57, 1151.

24. Rao, K. S., P. M. Krishna, D. M. Prasad, T. S. Latha, and C. Satyanarayana, 2008. Ind. J. Eng. Mater. Sci. 15, 215. 